\title{
ALGUNAS CONSIDERACIONES EN TORNO AL MODELO EDUCATIVO DE LA UNIVERSIDAD DE CHILE
}

\author{
SOME CONSIDERATIONS ABOUT THE EDUCATIONAL MODEL \\ OF THE UNIVERSIDAD DE CHILE
}

\author{
Juan Sebastián Vergara Palma \\ Departamento de Música \\ Facultad de Artes \\ Universidad de Chile
}

Resumen: Este artículo se estructura sobre la base de una observación crítica respecto de los alcances que tiene el Modelo Educativo de la Universidad de Chile, analizando tensiones producidas entre conceptos y los supuestos epistémicos que sostienen el engranaje de este modelo desde la perspectiva curricular. Asimismo, se tensionan las implicancias que tiene el modelo de formación basado en competencias y cómo éste se puede articular en función del paradigma humanista que posee esta universidad.

Palabras clave: humanismo, currículum, formación, selección, inclusión, acceso, aprendizaje.

Abstract: This article is structured on the basis of a critical observation regarding the scope of the Educational Model of the University of Chile, analyzing tensions produced between concepts and the epistemic assumptions that sustain the gearing of this model from the curricular perspective. Likewise, the implications of the competency-based training model and how it can be articulated according to the humanist paradigm that this university possesses are tense.

Key words: humanism, curriculum, training, selection, inclusion, access, learning.

\section{INTRODUCCIÓN}

El Modelo Educativo planteado por la Universidad de Chile se presenta dentro de un marco institucional muy permeado por la visión y misión que promueve y que, además, considera el contexto actual, caracterizado por "tensiones, desafíos y oportunidades sin precedentes" (Modelo Educativo, 2018). Asimismo, establece ciertos principios orientadores que permiten conducir los procesos formativos, como la formación integral de personas, la pertinencia educativa, la equidad e inclusión y la calidad educativa, tema muy en boga, hoy por hoy, en el sistema nacional de la educación terciaria.

En un primer análisis se aprecia una tensión producida entre la selección y la inclusión, es decir, por un lado se señala que la Universidad de Chile, dada su vocación de 
excelencia acoge a una población de elite intelectual, y por otro, abre sus puertas a toda la comunidad en su condición de universidad pública. El desafío que habría que plantear es en qué medida la Universidad de Chile se hace cargo de la brecha educativa por todos consabida y cómo concilia la tensión antes planteada, en un modelo que no resuelve esta problemática.

A modo de ejemplo, los estudiantes que ingresan a la carrera de Teoría de la Música, llegan de distintos establecimientos educacionales, con distinto capital cultural, dado que las realidades de donde provienen son muy disímiles, por cuanto no han tenido las mismas oportunidades de desarrollo ni en la formación humana a través de la música ni en la formación básica de la música a través de su práctica.

¿Es lícito plantear una selección para ingresar a la Universidad, en los temas artísticos, cuando no se han tenido las mismas oportunidades y por consiguiente, no se tienen las mismas condiciones? ¿Cuántas veces no se ha perseguido el prurito de encontrar grandes talentos?

Cuánto tiempo no se ha invertido en la tecnificación de los instrumentos evaluativos para la admisión a las carreras artísticas y en especial, a la música y no se ha reparado en que se les está cerrando las puertas a quienes nunca tuvieron una formación integral en el sistema escolar.

Pues bien, es necesario considerar el concepto de la inclusión como una vía para frenar el avance de la desigualdad. Si la Universidad de Chile, se ha comprometido al resguardo de la cultura en todas sus manifestaciones, debe pues incluir a todos sus ciudadanos en esta noble tarea.

En segundo término, siguiendo la misma línea de la inclusión, hay que señalar que falta declarar el tema de género, que, en conjunto con la cuestión del multiculturalismo, se inserta dentro de una perspectiva posmoderna y como tal, dentro de una realidad compleja multidimensional.

Por otra parte, ¿cómo se concibe un proceso formativo que tiene como propósito la formación humanista del sujeto, entre otros aspectos, naturalmente, y el sentido instrumental de la competencia, un constructo que proviene de la economía, condicionado por la empleabilidad? No se enfatiza en ese aspecto y quizás habría que plantear una suerte de re-significación de las competencias a la luz de una universidad compleja, con toda su historia y con todo su potencial.

Las demandas sociales no, necesariamente, son equivalentes a las demandas de la industria, que pretende condicionar la formación de los estudiantes en una dimensión de carácter instrumental, donde el saber hacer predomina por sobre el ser. Sobre este punto, el modelo educativo planteado, debe prever este tipo de matices y defender la necesidad de la formación del sujeto en tanto ciudadano que aporta y enriquece en un sentido 
integral: la Universidad asume con vocación de excelencia la formación de personas y la contribución al desarrollo espiritual y material de la Nación (Misión de la Universidad de Chile).

Luego, nos vemos enfrentados nuevamente a otra tensión, cual es la de la formación de nuestros estudiantes, la que a la luz de las supuestas demandas sociales, que, ciertamente, provienen de otro sector, pretende formar estudiantes en un sentido instrumental, atendiendo a una perspectiva unidimensional de la formación profesional, frente a los principios declarados en este modelo, que orientan la formación de personas, en primer término, lo que significa hablar desde una perspectiva multidimensional.

Esta problemática debiera plantearse también en este modelo, como parte de un conjunto de fenómenos que tensionan las fronteras entre "el ser" de una universidad que se declara profundamente humanista y el "deber ser" que plantea una sociedad, según sus propios intereses, permeada por conceptos como lo funcional y lo eficiente. Explicitar estas cuestiones es, explicitar la conciencia que se tiene de un escenario complejo multidimensional, en sintonía con el carácter reflexivo y crítico que caracteriza nuestra universidad.

Por último, se habla de principios y elementos estructurales del currículum así como también de los procesos de gestión y organización asociados, sin explicitar desde dónde se está entendiendo la base curricular, es decir, no se hace mención al fundamento teórico del currículum del cual emana el modelo educativo. Asimismo, respecto de la formación, se habla de las implicancias docentes pero no del rol del estudiante ni tampoco acerca de los enfoques, procesos, criterios y estrategias de la evaluación dentro de este modelo.

Si bien, se entiende que la propuesta es coherente con la misión, visión y los estatutos de nuestra casa de estudios, se hace necesario demostrar consistencia entre el modelo educativo propuesto y la teoría educativa que lo sustenta.

\section{LA CUESTIÓN DEL CURRÍCULUM}

El punto central de la observación de este modelo, es que manifiesta una formación basada en competencias, no enfatizando el concepto de competencia que adopta la Universidad de Chile. Se hace necesario, por lo menos consignar una definición operacional del término competencia, desde la óptica de una universidad compleja. Además, todos los elementos que subyacen al modelo planteado se describen en el plano más bien metodológico, teniendo que inferir la teoría y más aún las razones ontológicas que justifican este modelo.

El planteamiento de esta reflexión sugiere presentar un modelo más consistente en lo que respecta a la teoría del currículum, dejando en claro las bases en que se sustenta el Modelo Educativo que presenta la Universidad de Chile. En esa misma línea, no sería 
recomendable dejar abierta la posibilidad de supuestos que podría inducir a una lectura errada del pensamiento que hay detrás del modelo presentado, sobre todo, tomando en consideración la adopción de la formación por competencias no exenta de críticas. Autores como Scott y Aggar (en Barnett, 1994), con relación al concepto de la competencia y la técnica, entre otros por cierto, plantean la idea de la unidimensionalidad y el reduccionismo, en franca contradicción con la perspectiva de la casa de Bello. Otra vez, nos enfrentamos a una nueva tensión de orden ontológico - ideológico.

La idea de que la sociedad demande una formación más bien tecnificada a la universidad, es contraproducente en el sentido de que la generación del conocimiento debe propiciar un avance epistémico y no una fragmentación de éste, reduciendo todo conocimiento a un saber orientado hacia lo instrumental, en función de la empleabilidad. Es una realidad compleja, considerando que las acciones tienen un carácter bidireccional; la universidad se nutre de la sociedad pero también genera propuestas, las que deben ir en pro de una mejora de la sociedad misma, en el sentido humanista, según la visión y misión de nuestra universidad.

Dejando de lado la competencia e intentando descifrar la perspectiva que promueve este modelo educativo y con el afán de aportar, desde el plano ontológico se podría inferir que el paradigma educativo (optado por este modelo) es crítico - reflexivo ideológico, el cual tiene una visión global de la realidad, no exenta de condiciones ideológicas, económicas, políticas, históricas y sociales, articulada, generada y organizada en y desde la realidad, orientada hacia el cambio, la que supone una realidad mejorable. Adicionalmente se puede inferir una visión del mundo fenomenológico, subjetivo y holístico, orientado hacia los procesos, lo que implica comprender el comportamiento humano a partir del propio marco de referencia del individuo o sujeto; supone una realidad dinámica (Florez, 1995).

Por otra parte, desde el plano epistemológico, la Teoría Crítica del Currículum establece que los contenidos deben ser socialmente significativos, en que la educación se entiende como valoración consensuada, negociada, buscando evidencias de la contribución de las actividades a la mejora del contexto. En consecuencia, el profesor debe ser "un intelectual transformativo, crítico y reflexivo. Agente de cambio social y político" (Ruiz, 1996). En ese sentido, se puede entender el currículum como un proceso, en el que la interacción docente - estudiante le otorga "un sentido al conocimiento y a las acciones que se generan" (Romeo, 1998). Esto, ciertamente, se identifica con la formación humanista.

Luego, el fundamento teórico que sustenta el modelo podría estar entre la pedagogía constructivista y la pedagogía social. La primera, porque propone un diseño del currículo por procesos cognitivos, subjetivos e investigativos y la segunda porque considera el diseño del currículo por consenso participativo, es decir, supone la participación de toda la comunidad educativa (Florez, 1995). Se considera un punto intermedio porque tiene conceptos e ideas de ambas perspectivas; mientras la primera 
propone una enseñanza constructora de sentidos desde el estudiante, la segunda propone una enseñanza crítica y dialógica colectiva.

Con relación al proceso de enseñanza y aprendizaje, en el que claramente se define el foco centrado en el estudiante, se entiende el aprendizaje como fenómeno complejo, en que la experiencia juega un rol sustantivo, la construcción personal, es decir el auto aprendizaje, el carácter dialógico y social, entendido desde la interacción comunicativa y el carácter situado, el contexto. Esto sumado a que, si bien el currículum se organiza, desde la perspectiva de la educación basada en competencias, sobre la base de núcleos problemáticos, supone un cambio en el rol del docente como mediador y la interacción que de ello se desprende. Por consiguiente, se puede plantear esta modalidad como una operacionalización de la Teoría de la Modificabilidad Cognitiva Estructural de Feuerstein, la que releva la experiencia del aprendizaje mediado y el valor de la interacción humana. Asimismo, la Teoría Sociocultural de Vigotsky, le asigna un gran valor a la interacción social dentro del aprendizaje. Hoy por hoy, se considera la interacción como el foco de estudio.

Referente a los saberes, organizados en base a núcleos problemáticos, esta perspectiva plantea la idea de un saber contextualizado y mediado por múltiples factores, lejos de la fragmentación y el reduccionismo de la perspectiva de la técnica como procedimiento. El estudiante debe movilizar recursos de redes y de contexto para abordar un problema complejo. Este planteamiento se aproxima a la idea de una formación integral, superando la idea de la unidimensionalidad con que se ha venido trabajando los saberes o contenidos de una determinada asignatura o actividad curricular, dicho en los términos de una educación basada en competencias.

Respecto de las implicancias para la docencia, el modelo señala que "los procesos de enseñanza y aprendizaje se benefician cuando el trabajo reflexivo y colaborativo entre docentes se orienta a la revisión de las propias prácticas, a la investigación sobre la docencia, al intercambio de experiencias y a la generación de mejoras en los procesos formativos, para que todos los estudiantes logren aprendizajes significativos" (Modelo Educativo, 2018). A este respecto, la reflexión sobre la acción educativa sintoniza con la perspectiva curricular práctica, dado que “...la reflexión sobre la acción es un espacio insustituible en cualquier formación profesional. Se incentiva el análisis, inserto en la problematicidad del hacer concreto, desde una aproximación de diagnóstico de la realidad donde se opera, hasta los potenciales esquemas de pensamiento que subyacen en los contextos donde se desarrollan las acciones. Por tanto, formas de enseñanza vinculadas a la discusión crítica, a la búsqueda de los por qué, al planteamiento de hipótesis, a la interpretación de significados, a la redefinición de situaciones, a la reconfiguración de conceptos son propias... de la perspectiva práctica". (Romeo, 1998). 


\section{CONCLUSIONES}

El Modelo Educativo de la Universidad de Chile, dentro de sus planteamientos, en su afán por una formación de calidad, en un marco de equidad e inclusión, debe considerar y consignar aspectos tales como la tensión producida entre la selección y la inclusión, la tensión producida entre la formación integral del sujeto y el concepto de la competencia, la inclusión del tema de género en la propuesta educativa, el rol del estudiante en el modelo propuesto y la teoría curricular que sustenta el modelo educativo declarado.

Luego, enfatizar en que estas consideraciones en torno al modelo educativo apuntan, esencialmente, al fundamento curricular, el cual nos sitúa en una realidad dinámica, compleja, multidimensional.

La coherencia entre el plano ontológico, pasando por el epistemológico hasta llegar al metodológico, le da sentido y unidad estructural al currículum y todo modelo educativo no puede estar exento a este encadenamiento.

Los principios orientadores que plantea este modelo educativo, vale decir, la formación integral de personas, la pertinencia educativa, la equidad e inclusión y la calidad educativa, son, ciertamente, principios que subyacen de la visión y misión de la Universidad de Chile, sin embargo, se resiente cuando se plantea un modelo basado en competencias, sin enfatizar el sentido que se le da a dicha formación y al concepto de competencia asumido.

Finalmente, la tensión se concentra entre la lógica instrumental y los fines de la educación, en tanto formación del "ser" humano.

\section{REFERENCIAS BIBLIOGRÁFICAS}

Barnett, R. (1994) Los límites de la competencia. Barcelona: Editorial Gedisa.

Florez, R. (1999) Evaluación pedagógica y cognición. Bogotá: McGraw-Hill.

Romeo, J. (1998) Perspectivas del currículo en la educación superior. Revista Enfoques Educacionales 1(1).

Ruiz, J. (1996) Teoría del currículum. Madrid: Editorial Universitas, S.A.

Universidad de Chile. (2018) Modelo educativo. Santiago: Ediciones Universidad de Chile. 